\title{
ECM stiffness effects and subtumor formation in glioma growth. In silico model
}

\author{
Vladimir Kalinin \\ R\&D Sector of TMA, Dundalk, Ireland \\ vladimir.kalinin@tma-science.ie
}

\begin{abstract}
This study is based on in silico model and demonstrates how variations of Extracellular Matrix (ECM) stiffness may affect glioma invasive scenarios. The model describes cell proliferation and transport through elastic and compressible cell- ECM composite, including "go/grow" transitions determined by oxygen concentrations. Simulations generate invasive scenarios conditioned only by set of mechanical characteristics of cell-ECM composite typical for gliomas. Multicellular Tumor Spheroid (MTS) growing in soft matrix exhibits gentle cell density profile with elongated low density invasive zone, while rigid ECM conditions sharp edge of MTS and higher invasion speed. Moreover, the highest speed of tumor invasion is obtained at intermediate values of ECM stiffness, when a compact cell cluster is being formed instead of invasive zone as a second proliferative layer out of the core spheroid.
\end{abstract}

Keywords - Multicellular Tumor Spheroid, Extracellular Matrix, glioma invasiveness, cell adhesion, ECM stiffness, in silico model

\section{Introduction}

It has been found that purely mechanical characteristics of glioma microenvironment play pivotal role in the invasion process [1-4]. The importance of cell- ECM interaction and the role of matrix density, elasticity and porosity have emerged as a particularly strong regulators of migration and proliferation rate of malignant brain tumor cells $[2,3]$. Typically, the invasive properties of glioma grow up at increase of ECM stiffness [2-4]. Some cases however present exceptions, e.g. demonstrating biphasic function of rigiditydependence [3] or rigidity-insensitive high invasive capacity even in soft matrigel 3D cultures [4]. Therefore, in spite of general trends every case is unique regarding the behavior of each specific glioma cell line. The model used in the current study is not intended to simulate these unique cases. It does not take into account individual glioma cell line attributes and therefore cannot reproduce the variety of invasive patterns typically exhibited by different gloma cell lines [5]. Based on average glioma cell characteristics, the model generates "first approximation" scenarios of glioma invasion invariable for all glioma cell lines. These basic scenarios do not involve signaling regulation of glioma, but present a useful starting point for further analysis of every unique case. The in silico model describes visco-elastic dynamics of cellsECM composite and is capable to simulate the interplay between elastic cell- ECM interaction, cell- cell adhesion and cell phenotype transition in the frame of "go/grow" approach [6]. The simulations aimed at studying glioma MTS growth as it depends on ECM stiffness.

\section{Model assumptions}

Spherically symmetric 1D simulation domain contains two components: cells and ECM as constituents of a composite system. ECM is considered to be purely elastic compressible scaffold, while the compressible cell aggregation exhibits viscoelastic properties and also effects of cell-cell adhesion. The total stress distribution in cellECM composite build the driving forces for cell transport. There are three cell phenotypes considered in the model following soft version of go/grow scenario [6]: proliferative cells that can proliferate and have low, non- zero, motility; hypoxic cells that have low, non- zero, proliferation rate and exhibit high motility, and necrotic cells, which are inert- do not exhibit motility and do not proliferate. Diffusion of oxygen towards the spheroid center from the spherical domain border and its local consumption determine the distribution of oxygen concentration along with phenotype transition rates. The model is formulated in more details in [7], including initial and boundary conditions. Model validation has been carried out on the results of two in vitro studies of glioma MTS growth [1,8] and exhibited rather good agreement with the experimental data, see [7].

\section{Effect of matrix stiffness}

A series of MTS growth calculations have been carried out at variation of ECM (collagen) stiffness from $\mathrm{E}_{\mathrm{ECM}}=$ $500 \mathrm{~Pa}$ to $5 \mathrm{kPa}$. All calculations demonstrate a presence of Transition Layer (TL) on spheroid surface, where MTS mechanical characteristics undergo a sharp transition. The sharp raise of the total stress across the transition layer builds hydrostatic pressure gradients within this narrow area directed outwards across the TL. Therefore, TL represents a potential barrier for motile cells motion out of MTS core. The resulting pressure force steepens the front of cell density distribution, pushing back some of the motile cells traveling out of the core. The stress barrier of TL depends monotonically on ECM stiffness. So, that MTS growth in relatively soft collagens of $\mathrm{E}_{\mathrm{ECM}} \sim 500 \mathrm{~Pa}$ [8] exhibits typically thin TL and elongated invasive zone around the core spheroid with rarefied cell population in it $[7,8]$. The stiff matrix of $\mathrm{E}_{\mathrm{ECM}}=2.5 \mathrm{kPa}$ generates tenfold higher stresses within TL. This difference can be easily seen on peak magnitudes of the radial stress component $\sigma_{\text {Rtot }} / \sigma_{0}$ comparing TLs on Fig. 1a with the data obtained in [7] for $\mathrm{E}_{\mathrm{ECM}}=500 \mathrm{~Pa}$. The counteraction of rigid matrix against expanding cell aggregation forms a sharp front at its edge without elongation of cell density profile into peripheral zone typical for soft matrixes, see [7]. The simulations show monotonic decrease of average spheroid size at increase of ECM stiffness along with growth of its expansion speed.

\section{Formation of subtumors}

Another interesting effect of ECM stiffness variations is a discrete form of invasive zone obtained at $\mathrm{E}_{\mathrm{ECM}}=2.5 \mathrm{kPa}$. The increase of matrix stiffness reduces concentration of motile cells coming through TL barrier and ultimately leads to contraction of invasive zone into a second narrow proliferative layer outside of the core spheroid (Fig.1). 

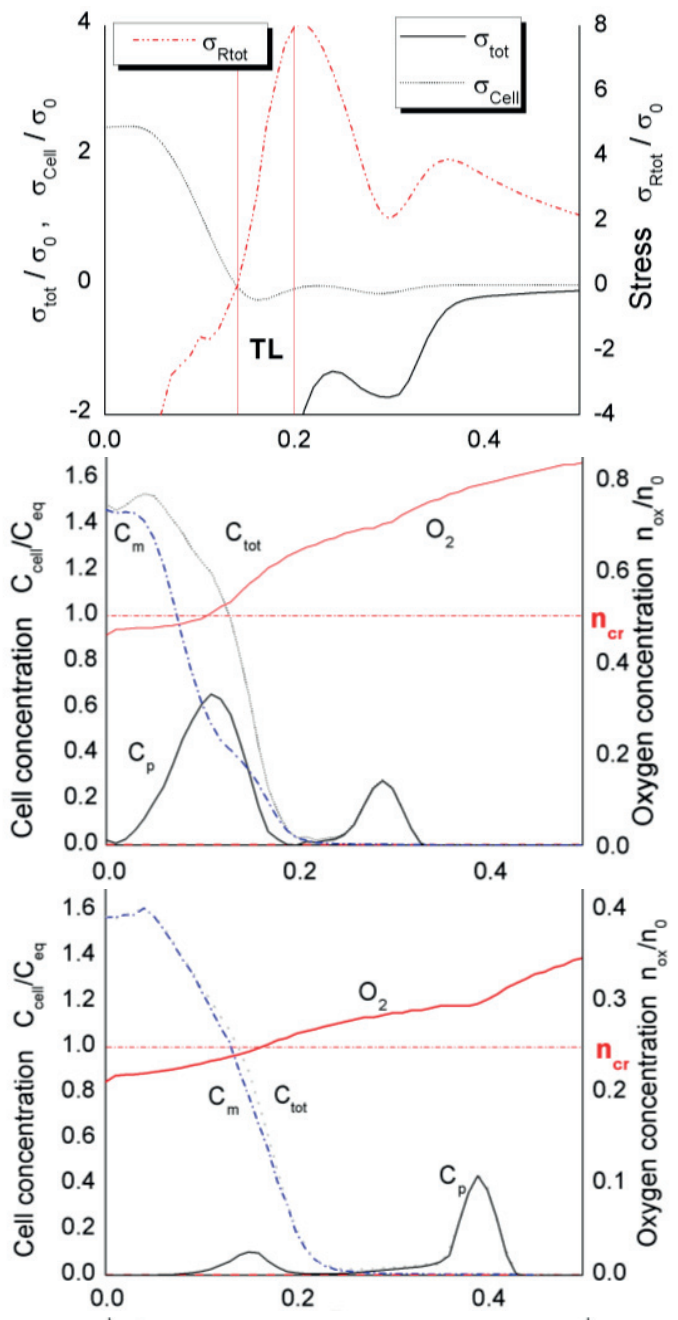

b)

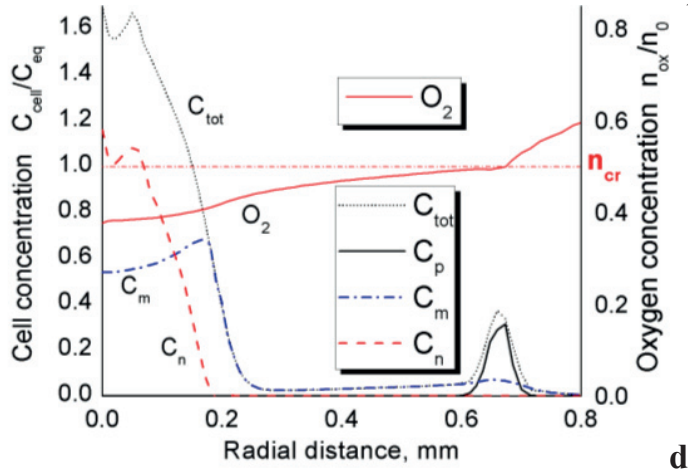

)

d)

Fig. 1. Formation of Subtumor days 2, 3 and 5 of MTS growth at elevated matrix stiffness $\mathrm{E}_{\mathrm{ECM}}=2.5 \mathrm{kPa}$ : (a) stress distribution for day 2 including total stress in cells-ECM composite $\sigma_{\text {tot }}$, intercellular stress distribution $\sigma_{\text {cell }}$ and radial component of the total stress $\sigma_{\text {Rtot. }} ; \sigma_{0}=100 \mathrm{~Pa} ;(\mathrm{b}, \mathrm{c}, \mathrm{d})$ radial cell density distributions for proliferative cells $C_{p}$, hypoxic $C_{m}$, necrotic $C_{n}$ and total cell density $\mathrm{C}_{\text {tot }} ; \mathrm{C}_{\text {eq }}=3.5 \times 10^{8}$ cells $/ \mathrm{cm}^{3}$. Distribution of oxygen concentration is also shown on these graphs; the dotted line $\mathrm{n}_{\mathrm{cr}}$ shows the threshold of $\mathrm{O}_{2}$ level for proliferative-to-hypoxic cell phenotype transition, $\mathrm{n}_{0}=4 \mathrm{mg} / \mathrm{L}$;

By day two shown on the figure, the major part of the main proliferative layer is located in the area of oxygen shortage and undergoes cell phenotype transition, while another small layer is formed out of the main one. Cell- cell adhesion and rigid matrix keep this layer compact instead of elongated invasive zone typical for soft matrixes. A radial component of the total stress in cell- ECM system exhibits strong gradient on the inner side of the newly formed layer (fig.1a) pushing it out of the core spheroid. By day three (fig.1c) over $90 \%$ of the main proliferative rim turns hypoxic, as the oxygen level inside the core part drops below the transition threshold (fig.1c). By day 5, the simulation shows that only the newly formed distant cluster of cells is out of the large hypoxic area, while the core spheroid turns hypoxic with growing necrotic zone in its center. Interestingly, the MTS does not form a distant proliferative layer at higher $\mathrm{ECM}$ stiffness $\mathrm{E}_{\mathrm{ECM}}=5 \mathrm{kPa}$ forming only a sharp edge of the core spheroid. The tumor invasion speed in our simulations exhibits biphasic function of $\mathrm{E}_{\mathrm{ECM}}$ with its maximum around $\mathrm{E}_{\mathrm{ECM}} \sim 2.5 \mathrm{kPa}$, when the distant proliferative layers are being formed.

\section{Conclusions}

Three scenarios of glioma invasion at different ECM stiffness values, driven by mere mechanical principles of continuum and iso-strain dynamics of composite system have been shown in simulations. These scenarios may contribute into glioma variability in vivo being mediated within signaling feedback loop of self-consistent glioma regulation.

\section{REFERENCES}

[1] V.D. Gordon, M.T. Valentine, M.L. Gardel et al., " Measuring the mechanical stress induced by an expanding multicellular tumor system: a case study", Experimental Cell Research 289, 2003, pp.58 66.

[2] B. Ananthanarayanan, Y. Kim, S. Kumar, "Elucidating the mechanobiology of malignant brain tumors using a brain matrixmimetic hyaluronic acid hydrogel platform," Biomaterials,_32(31), 2011, pp.7913-23.

[3] T.A. Ulrich, E. de Juan Pardo, S. Kumar, "The mechanical rigidity of the extra-cellular matrix regulates the structure, motility, and proliferation of glioma cells", Cancer Res, 69(10), 2009, pp. 4167-74.

[4] T.J. Grundy, E. de Leon, K.R. Griffin et al., "Differential response of patient-derived primary glioblastoma cells to environmental stiffness", Scientific Reports 6, 23353, 2016.

[5] M.Vinci, C.Box, S.A. Eccles, "Three-Dimensional (3D) Tumor Spheroid Invasion Assay", Journal of Visualized Experiments (99), e52686, 2015.

[6] H. Hatzikirou, D. Basanta, M. Simon et al.,"Go or grow': The key to the emergence of invasion in tumor progression?", Mathematical Medicine and Biology, 29(1), 2012, pp. 49-65

[7] V. Kalinin, "In Silico model of glioma MTS growth. Effects of compression and mechanical ECM remodeling", 'Mathematical methods and high-performance computing in the life sciences, biomedicine and biotechnology' at 12th Int.Multiconf. BGRS/SB2020, 06-10 July 2020, Novosibirsk, Russia

[8] A.Stein, T. Demuth, D.Mobley et al.,, "A mathematical model of glioblastoma tumor spheroid invasion in a three-dimensional in vitro experiment," Biophysical Journal, 92(1), 2007, pp. 356-365. 